\title{
Bifurcation and chaos in a dc-driven long annular Josephson junction
}

\section{Grnbech-Jensen, N.; Lomdahl, Peter S.; Samuelsen, Mogens Rugholm}

\section{Published in:}

Physical Review B

Link to article, DOI:

10.1103/PhysRevB.43.12799

Publication date:

1991

Document Version

Publisher's PDF, also known as Version of record

Link back to DTU Orbit

Citation (APA):

Grnbech-Jensen, N., Lomdahl, P. S., \& Samuelsen, M. R. (1991). Bifurcation and chaos in a dc-driven long annular Josephson junction. Physical Review B, 43(16), 12799-12803.

https://doi.org/10.1103/PhysRevB.43.12799

\section{General rights}

Copyright and moral rights for the publications made accessible in the public portal are retained by the authors and/or other copyright owners and it is a condition of accessing publications that users recognise and abide by the legal requirements associated with these rights.

- Users may download and print one copy of any publication from the public portal for the purpose of private study or research.

- You may not further distribute the material or use it for any profit-making activity or commercial gain

- You may freely distribute the URL identifying the publication in the public portal 


\title{
Bifurcation and chaos in a dc-driven long annular Josephson junction
}

\author{
N. Grønbech-Jensen* and P. S. Lomdahl \\ Theoretical Division and Center for Nonlinear Studies, Los Alamos National Laboratory, \\ Los Alamos, New Mexico 87545 \\ M. R. Samuelsen \\ Physics Laboratory I, The Technical University of Denmark, DK-2800 Lyngby, Denmark
}

(Received 5 February 1991)

\begin{abstract}
Simulations of long annular Josephson junctions in a static magnetic field show that in large regions of bias current the system can exhibit a period-doubling bifurcation route to chaos. This is in contrast to previously studied Josephson-junction systems where chaotic behavior has primarily been observed in an external ac field.
\end{abstract}

Recently there has been some interest in the subject of chaotic behavior of soliton motion in the perturbed sineGordon system. ${ }^{1-7}$ This system has been used to describe many physical objects in one-dimensional problems, such as charge-density waves, dislocations in solids, nonlinear spin waves in superfluid phases of ${ }^{3} \mathrm{He}$, ferromagnetic or antiferromagnetic systems, etc. In particular the perturbed sine-Gordon system has been successful in explaining and predicting the behavior of flux flow in long Josephson tunnel junctions (JTJ's). ${ }^{8}$ Studies of the dynamics of magnetic fluxons in long Josephson junctions coupled to external magnetic fields have been a subject undergoing much progress in the past few years. The modeling has mostly been done in terms of soliton solutions to the perturbed sine-Gordon equation. In the case of oscillating rf magnetic fields, studies have been made to understand the phase-locking phenomena observed experimentally. $9,6,10$ In the case of constant dc magnetic fields, work has been done to explain the so-called Fiske steps in the dc current-voltage characteristics of long linear JTJ's. ${ }^{3-5,11}$ In both cases the interaction between the junction and the magnetic field has typically been modeled as perturbations of the open boundary conditions to the finite-size perturbed sine-Gordon system. However, recently it was demonstrated that under some circumstances this interaction could also take place in the interior of the system. ${ }^{12}$

The aim of this paper is to demonstrate that the fluxon motion in a junction, with annular geometry coupled to an external dc magnetic field, can result in bifurcations and chaotic motion. This is in close analogy with the reported chaotic and intermittent behavior of the Fiskestep mode. ${ }^{3-5}$ However, there are some significant differences between the system studied here and the one studied in Refs. 3-5, since the annular geometry does not provide any open boundary conditions through which the external magnetic field can perturb the system. Also, the Fiske-step mode of the long linear junction is characterized by an infinite sequence of annihilation and creation of solitons, whereas the annular geometry junction fixes the total number of solitons as a constant in time.
The perturbed sine-Gordon system studied here is given by $^{8,12}$

$$
\phi_{x x}-\phi_{t t}-\sin (\phi)=\Delta \frac{\partial}{\partial x}(\mathbf{B} \cdot \mathbf{n})+\alpha \phi_{t}-\eta .
$$

In order to fix the total number of solitons to one, the boundary conditions for the annular geometry are chosen to be

$$
\phi(0)=\phi(L)-2 \pi .
$$

Here $\mathbf{B}$ is the normalized magnetic field, the normalized flux density of the junction is $\phi_{x}$ with orientation $\mathbf{n}$, where $\mathbf{n}$ is a unit vector, and $\Delta$ is the coupling between the external field $\mathbf{B}$ and the flux density of the junction. For a JTJ which consists of two superconductors separated by a thin insulating layer, $\phi$ denotes the quantummechanical phase difference between the wave functions of the two superconductors, and the normalized spacedependent voltage across the thin barrier is proportional to $\phi_{t} \cdot{ }^{8}$ The normalized loss due to quasiparticle tunneling is given by $\alpha \phi_{t}$ and the normalized bias current density forced through the junction is given by $\eta$. The spatial dimension $(x)$ is normalized to the Josephson penetration depth $\lambda_{J}$ and the time dimension $(t)$ is normalized to the inverse plasma frequency $\omega_{0}^{-1}$ of the junction.

If we consider the magnetic field as spatially homogeneous we get the following equation of motion from Eq. (1):

$$
\phi_{x x}-\phi_{t t}-\sin (\phi)=b \sin (k x)+\alpha \phi_{t}-\eta,
$$

where $b=B \Delta 2 \pi / L$ and the wave number $k=2 \pi / L$ corresponds to the chosen length of the system. Defining the total energy $H$ of the system Eq. (3) as

$$
H=\int_{0}^{L}\left(\frac{1}{2} \phi_{x}^{2}+\frac{1}{2} \phi_{t}^{2}+1-\cos \phi\right) d x,
$$

we get the power input to the system as

$$
\frac{d H}{d t}=-\alpha \int_{0}^{L} \phi_{t}^{2} d x+\eta \int_{0}^{L} \phi_{t} d x-b \int_{0}^{L} \phi_{t} \sin (k x) d x .
$$

The soliton solution to the unperturbed sine-Gordon sys- 
tem [left-hand side of Eq. (3)] with infinite length is given by

$$
\phi=4 \tan ^{-1}\left\{\exp \left[\gamma(u)\left(x-x_{0}-u t\right)\right]\right\},
$$

where the instantaneous soliton position is $x_{0}$, the velocity is $u$ and the inverse Lorentz contraction is $\gamma(u)$ $=1 /\left(1-u^{2}\right)^{1 / 2}$. Using the adiabatic perturbation treatment ${ }^{8}$ we insert the profile Eq. (6) in Eq. (4) and Eq. (5) and get

$$
\begin{aligned}
& H=8 \gamma(u) \\
& \frac{d H}{d t}=-8 \alpha u^{2} \gamma(u)-2 \pi \eta u \\
& \quad+2 \pi u b \operatorname{sech}\left(\frac{\pi^{2}\left(1-u^{2}\right)^{1 / 2}}{L}\right) \sin \left(k x_{0}\right) .
\end{aligned}
$$

As noted in Ref. 12 we can then find a zero-voltage state $(u=0)$ if

$$
|\eta| \leq b \operatorname{sech}\left(\frac{\pi^{2}}{L}\right) \sin \left(k x_{0}\right) .
$$

Hence, by variation of the soliton position $x_{0}$ we find the largest possible bias current of a zero-voltage (-velocity) state to be

$$
|\delta \eta|=b \operatorname{sech}\left(\frac{\pi^{2}}{L}\right) .
$$

In the low-velocity limit $\left(u^{2}<<1\right)$ we get the equation of motion from Eq. (8)

$$
\frac{d^{2} \psi}{d t^{2}}=-\alpha \frac{d \psi}{d t}-\frac{\pi \eta k}{4}+\frac{\pi k}{4}|\delta \eta| \sin (\psi),
$$

where $\psi=k x_{0}$. This is the equation of motion for the damped and driven pendulum. The analogy between a small Josephson junction ${ }^{13}$ without any spatial extension and the annular long junction coupled to an external dc magnetic field is now clear, since $\psi$ corresponds to the quantum-mechanical phase difference between the two superconductors of a small junction with the critical current $|\delta \eta|$. However, in the high-velocity limit, Eq. (8) reduces to

$$
\frac{d \psi}{d t}=\frac{\pi \eta / 4 \alpha}{\left[1+(\pi \eta / 4 \alpha)^{2}\right]^{1 / 2}}
$$

which is equivalent to the power balance velocity of the soliton in the long junction with no magnetic field. Hence, the close analogy with the small junction is then only true in the low-velocity limit, since the $I-V$ characteristics of the annular junction will approach an almost vertical asymptote, corresponding to the largest velocity of the soliton $(u \approx 1)$, as the bias current is increased to $\eta \approx 1$. For bias currents $\eta>1$, there will be no localized $2 \pi$ kink and the system will behave similarly to the small junction. ${ }^{14}$

The results of the numerical solution of Eq. (3) are shown for the system parameters $L=6, \alpha=0.075$, and $b=0.75$. The numerical method used is a finitedifference scheme, second order in time and fourth order in space. In Fig. 1(a) we show the calculated dc $I$ - $V$ curve of the system. Initially the bias current $\eta$ was chosen to be zero. For $\eta$ very close to the value $|\delta \eta| \approx 0.279$ of Eq. (10) we find that the junction switches to a state with finite voltage, roughly given by $\left\langle\phi_{t}\right\rangle \approx 2 \pi u / L$, where $u$ is given by Eq. (12). Decreasing the bias current below the value $|\delta \eta|$ we stay on a finite-voltage branch of the $I-V$ curve until the system switches back to the zero-voltage state at about $\eta \approx 0.073$. However, a large part of the shown curve consists of states much more complicated than the behavior predicted by the adiabatic perturbation results Eqs. (10)-(12). The two resonantlike steps visible in Fig. 1(a) have their origin in the more detailed behavior shown in Fig. 1(b). Here we have plotted the time of flight for the soliton to pass one time through the junction. Clearly, we observe a bifurcation cascade corresponding to each of the two steps in the $I-V$ curve Fig. 1(a). In both sequences of bifurcations Fig. 1(b) we find hysteresis as indicated also in Fig. 1(a). To make sure we
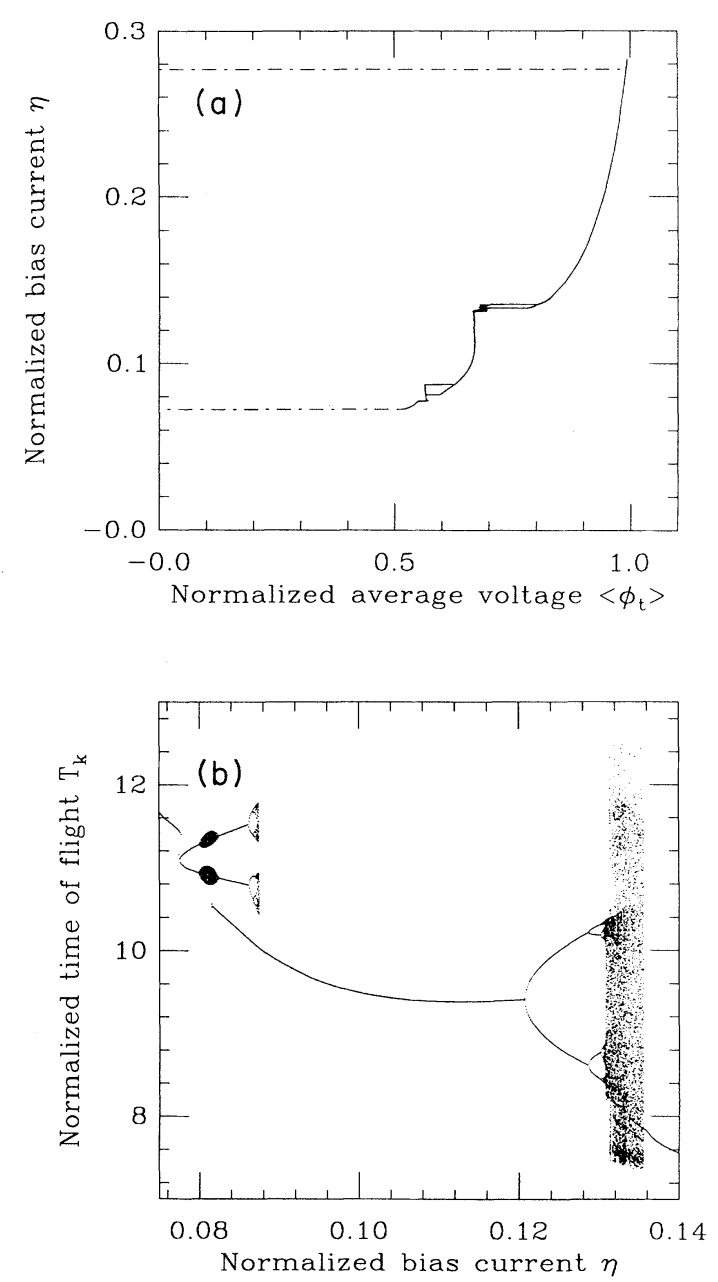

FIG. 1. Solution of Eq. (3) under variation of the normalized bias current $\eta$ for the system with the normalized parameters: $\alpha=0.075, b=0.75$, and $L=6$. (a) Part of the normalized dc $I-V$ curve. (b) Poincare-like plot showing the time of flight for the soliton to pass one time through the junction. 
have reached a steady state we have allowed the system a transient time of 8000 normalized time units after each change of $10^{-4}$ in the normalized bias $\eta$.

In Fig. 2 we show color contour plots of the normalized magnetic flux density $\phi_{x}$ as a function of $x$ and $t$ for three different values of the normalized bias current $\eta$. The time axes are horizontal and cover a time interval of 200 normalized time units. The space axes are vertical, covering the junction length of 6 normalized units. The color code, indicating the value of $\phi_{x}$, is shown to the left, where the low value (black) corresponds to $\phi_{x}=-1$ and the high value (yellow) to $\phi_{x}=5$.

The bottom plot, Fig. 2(a), shows the system dynamics for $\eta=0.115$. Here we see the origin of the steplike structure in the dc $I-V$ curve Fig. 1 . The soliton motion through the junction is perturbed by the spatially oscillat- ing background, and the soliton motion excites timedependent background modes, which are then interacting with the soliton motion. Although we have a soliton $(2 \pi$ kink) present at all times, this strong interaction between soliton and background is very similar to the Fiske-step mode in long linear junctions coupled to external magnetic fields in which a soliton annihilation at one boundary creates a plasma wave which then triggers a soliton creation at the other boundary. Figure 2(b) shows the system for $\eta=0.125$. Here the period-two behavior is visible as expected from Fig. 1(b). Increasing $\eta$ further the system passes through a period-doubling sequence and for $\eta=0.135$ we find [Fig. 2(c)] the system in chaotic motion with irregular behavior of the soliton trace (red and yellow) and the background. The bias value $\eta=0.135$ is in the hysteresis region, seen in Fig. 1(a), and

(d)

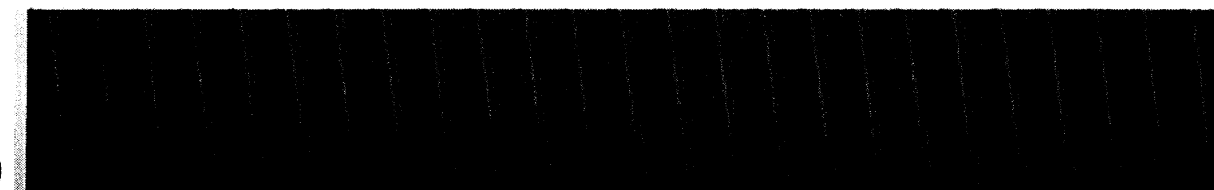

\section{(d)}

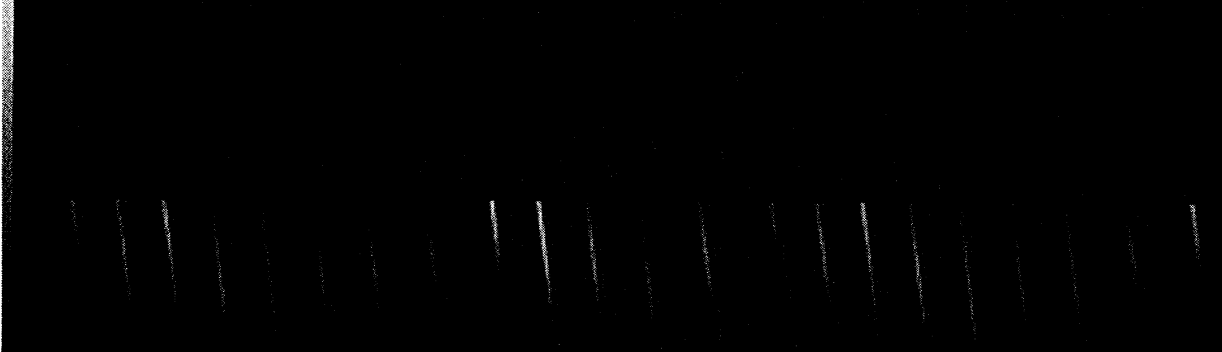

(c)

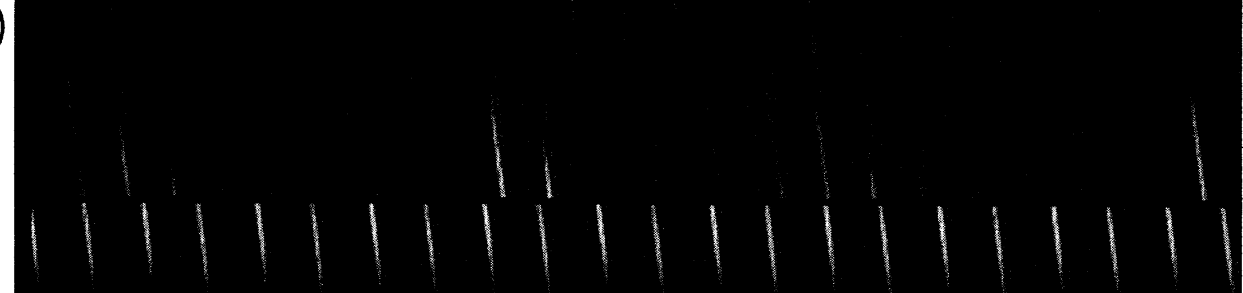

(b)

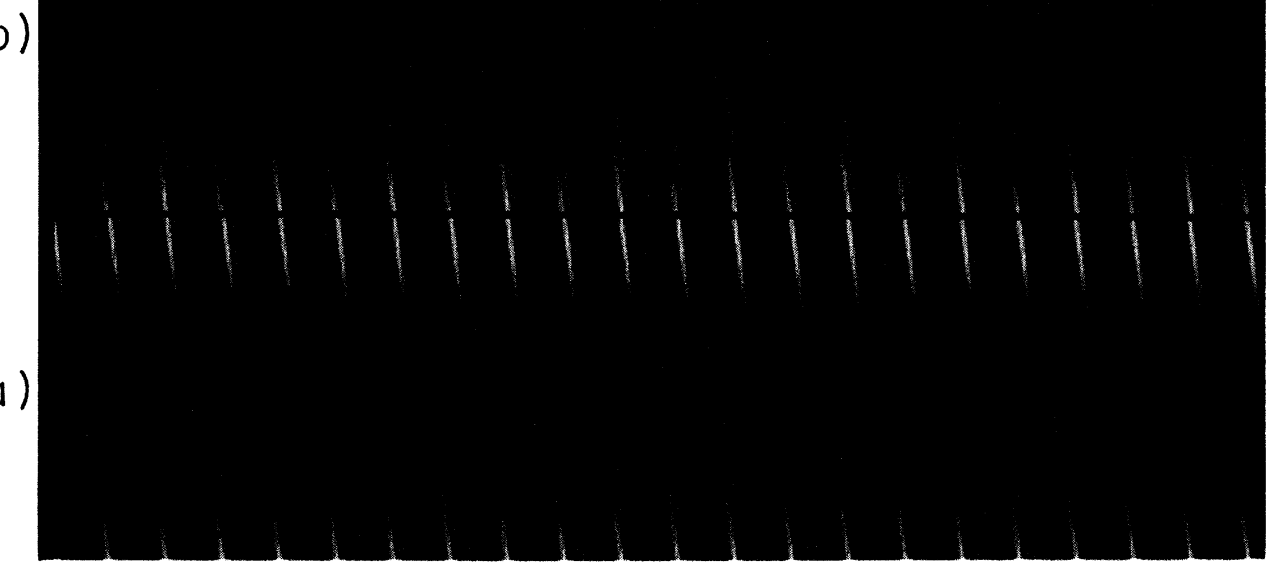

FIG. 2. Contour plots of the normalized flux density $\phi_{x}$ as a function of space and time. The color code is shown to the left as a vertical bar indicating the lowest value $\phi_{x}=-1$ as black and the largest value $\phi_{x}=5$ as yellow. The parameters are as in Fig. 1. (a) (bottom plot) Period one for $\eta=0.115$. (b) (next to bottom plot) Period two for $\eta=0.125$. (c) (next to top plot) Chaotic for $\eta=0.135$. (d) (top plot) Period one for $\eta=0.135$. 
Fig. 2(d) shows the system with the same parameters as Fig. 2(c), but in the state on the nonchaotic, highvoltage, branch. This state is nearly independent of the external magnetic field, since the soliton trace is almost a straight line unlike the traces in Figs. 2(a), 2(b), and 2(c). Here it is also interesting to note the differences between
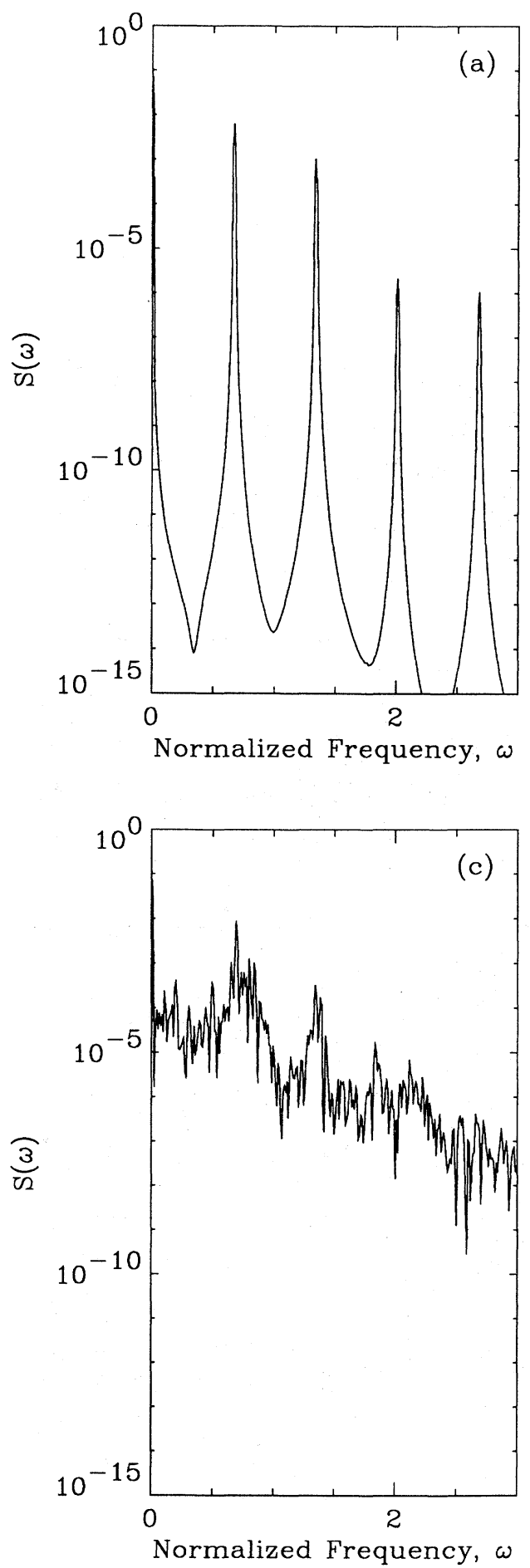

the modes on the steplike states [near vertical parts of the $I$ - $V$ curve Fig. 1(a)] and the modes above the steps. Comparing Fig. 2(a) with Fig. 2(d) we find that even though Fig. 2(a) shows a situation with lower bias (and lower average soliton velocity) than Fig. 2(d) we find that near $x=0$ and $x=L$ the soliton moves faster (high $\phi_{x}$ ) than at
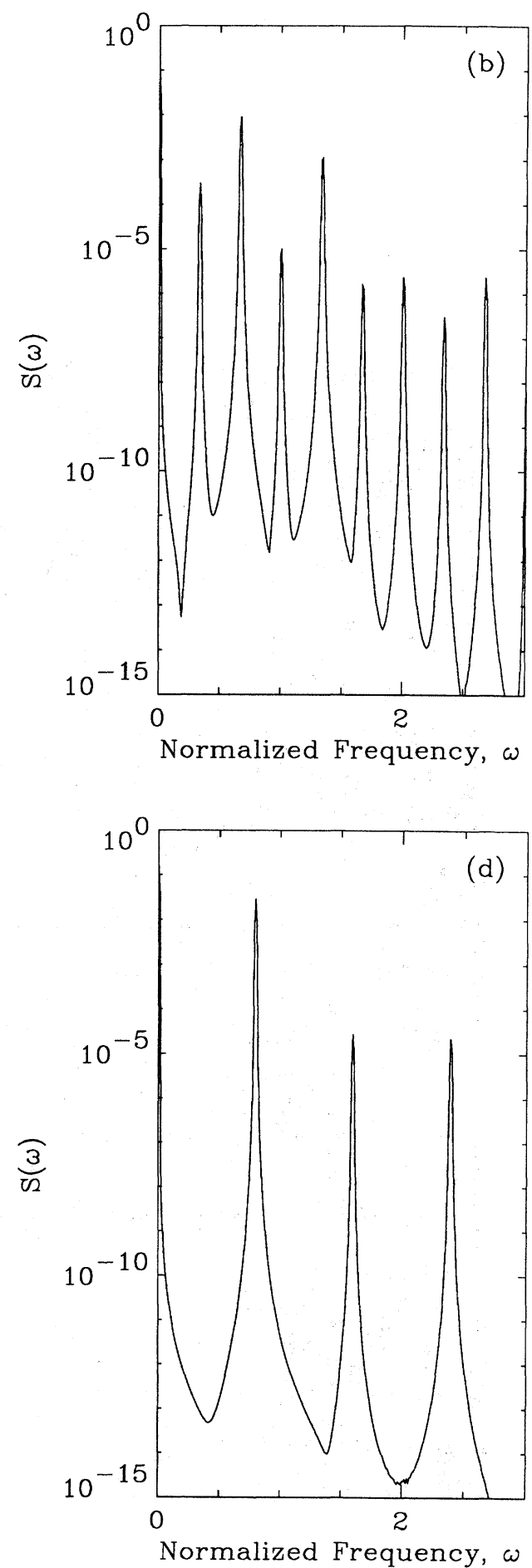

FIG. 3. Normalized spectral power density $S(\omega)$ of the spatially averaged junction voltage $\mathcal{V}=L^{-1} \int_{0}^{L} \phi_{t} d x$ for the four situations shown in Fig. 2. (a) Period one for $\eta=0.115$. (b) Period two for $\eta=0.125$. (c) Chaotic for $\eta=0.135$. (d) Period one for $\eta=0.135$. 
any point in Fig. 2(d). This is, of course, compensated by the very slow soliton motion $\left(\right.$ small $\left.\phi_{x}\right)$ near $x=L / 2$ in Fig. 2(a), resulting in lower average velocity (average voltage) than the state shown in Fig. 2(d). The steplike structures and the bifurcation cascades leading to chaos are caused by the interaction between the soliton mode and some excited cavity modes, which act like a feedback to the soliton mode. Hence we do not believe that this kind of complicated behavior is present in the simplified collective coordinate approach, which models the system as a relativistic particle (the soliton) in a constant spatially periodic potential [Eq. (8)].

Finally we show in Fig. 3 the spectral power density $S(\omega)$ of the normalized (spatially averaged) voltage $\mathcal{V}=L^{-1} \int_{0}^{L} \phi_{t} d x$ for the modes shown in Fig. 2. Figure 3(a) shows the spectrum of the period-one mode in Fig. 2(a) for $\eta=0.115$. The fundamental power contribution at $\omega=\omega_{s} \approx 0.65$ is seen and in addition three harmonics. For $\eta=0.125$ we find a considerable subharmonic generation at $\omega=\frac{1}{2} \omega_{s}$ corresponding to the bifurcated state Fig. 2(b). Figures 3(c) and 3(d) show the spectra of the two different modes for $\eta=0.135$. Fig. 3(c) is clearly representing the chaotic mode, since we observe a large noise rise at all frequencies, whereas Fig. 3(d) represents the periodic mode of Fig. 2(d).

The dynamical states in a long annular Josephson junction and a long linear junction have been found to be very similar if the systems are coupled to an external static magnetic field. In spite of the direct differences between the Fiske-step mode in linear junctions and the type of mode studied in this paper, we find that both systems are able to make bifurcation cascades and to operate in chaotic modes. Like the observations ${ }^{3-5}$ of the negative dynamical resistance branch in the dc $I-V$ curves, we observe a weak indication of negative dynamical resistance in the bias region between $\eta=0.11$ and $\eta=0.13$ (the negative resistance branch is more clear if a choice of $\alpha=0.1$ is made). However, unlike the observations ${ }^{3-5}$ done at the Fiske-step mode, we have been able to observe this feature in a nonchaotic and nonbifurcated branch of states.

We finally note that long annular Josephson junctions are well within current fabrication capabilities. ${ }^{15-17}$ Measurements have been made on these systems containing one or more solitons. The bifurcation and chaos phenomena described in this paper should therefore be easy to verify experimentally.

One of the authors (N.G.J.) is grateful to Otto Mønsteds fond and to Christian og Ottilia Brorsons Rejselegat for financial support. We also thank the Los Alamos Advanced Computing Laboratory for generously allowing access to their facilities. This work was performed under the auspices of the U.S. Department of Energy.
*Permanent address: Physics Laboratory I, The Technical University of Denmark, DK-2800 Lyngby, Denmark.

${ }^{1}$ A. R. Bishop, K. Fesser, P. S. Lomdahl, W. C. Kerr, M. B. Williams, and S. E. Trullinger, Phys. Rev. Lett. 50, 1095 (1983).

${ }^{2}$ A. R. Bishop and P. S. Lomdahl, Physica D 18, 54 (1986).

${ }^{3}$ M. P. Soerensen, N. Arley, P. L. Christiansen, R. D. Parmentier, and O. Skovgaard, Phys. Rev. Lett. 51, 1919 (1983).

${ }^{4}$ D. J. Zheng, W. J. Yeh, and O. G. Symko, Phys. Lett. A 140, 225 (1989).

${ }^{5}$ W. J. Yeh, O. G. Symko, and D. J. Zheng, Phys. Rev. B 42, 4080 (1990).

${ }^{6}$ M. Salerno, M. R. Samuelsen, G. Filatrella, S. Pagano, and R. D. Parmentier, Phys. Rev. B 41, 6641 (1990).

${ }^{7}$ M. Salerno, Phys. Lett. A 144, 453 (1990).

${ }^{8}$ D. W. McLaughlin and A. C. Scott, Phys. Rev. A 18, 1652 (1978).

${ }^{9}$ M. Salerno, M. R. Samuelsen, G. Filatrella, S. Pagano, and R.
D. Parmentier, Phys. Lett. A 137, 75 (1989).

${ }^{10}$ N. F. Pedersen and A. Davidson, Phys. Rev. B 41, 178 (1990).

${ }^{11}$ B. Dueholm, E. Joergensen, O. A. Levring, J. Mygin, N. F. Pedersen, M. R. Samuelsen, O. H. Olsen, and M. Cirillo, Physica B + C 108B, 1303 (1981).

${ }^{12}$ N. Grønbech-Jensen, P. S. Lomdahl, and M. R. Samuelsen, Phys. Lett. A 154, 14 (1991).

${ }^{13}$ See, e.g., A. Barone and G. Paterno, Physics and Applications of the Josephson Effect (Wiley, New York, 1982).

${ }^{14}$ S. Pagano, M. P. Soerensen, R. D. Parmentier, P. L. Christiansen, O. V. Skovgaard, J. Mygind, N. F. Pedersen, and M. R. Samuelsen, Phys. Rev. B 33, 179 (1986).

${ }^{15}$ A. Davidson, B. Dueholm, B. Kryger, and N. F. Pedersen, Phys. Rev. Lett. 55, 2059 (1985).

${ }^{16}$ A. Davidson and N. F. Pedersen, Appl. Phys. Lett. 44, 465 (1983).

${ }^{17}$ A. Davidson, B. Dueholm, and N. F. Pedersen, J. Appl. Phys. 60, 1447 (1986). 


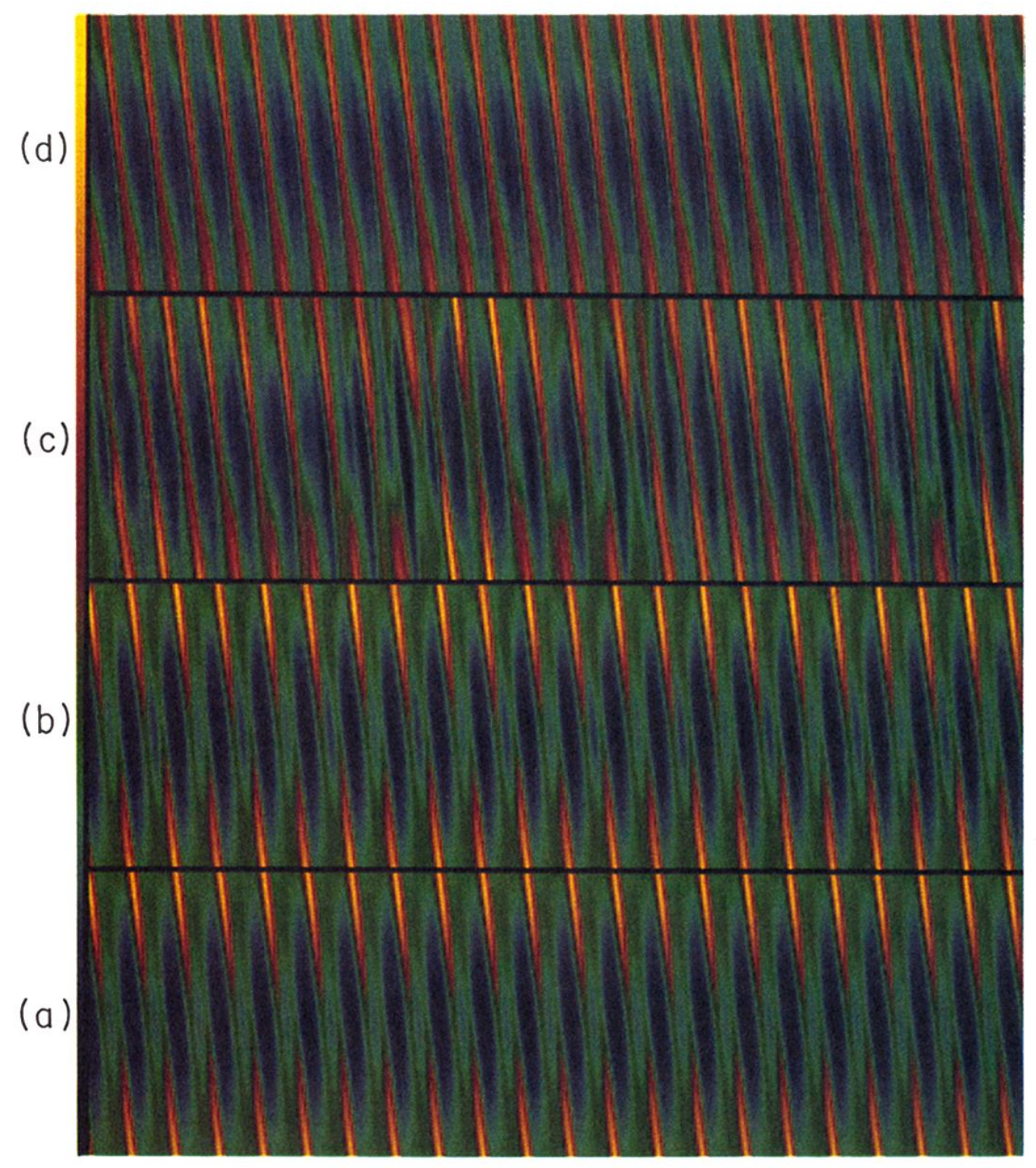

FIG. 2. Contour plots of the normalized flux density $\phi_{x}$ as a function of space and time. The color code is shown to the left as a vertical bar indicating the lowest value $\phi_{x}=-1$ as black and the largest value $\phi_{x}=5$ as yellow. The parameters are as in Fig. 1 . (a) (bottom plot) Period one for $\eta=0.115$. (b) (next to bottom plot) Period two for $\eta=0.125$. (c) (next to top plot) Chaotic for $\eta=0.135$. (d) (top plot) Period one for $\eta=0.135$. 